\title{
Time-resolved Cathodoluminescence in a Transmission Electron Microscope Applied to NV Centers in Diamond
}

Sophie Meuret ${ }^{1}$, Yves Auad ${ }^{2}$, Luiz Tizei ${ }^{3}$, H.C. Chang ${ }^{4}$, Florent Houdellier ${ }^{1}$, Mathieu Kociak ${ }^{3}$ and Arnaud Arbouet ${ }^{1}$

${ }^{1}$ CEMES/CNRS, Toulouse, Midi-Pyrenees, France, ${ }^{2}$ Laboratoire de Physique des Solides, Orsay, Ile-deFrance, France, ${ }^{3} \mathrm{CNRS}$, Orsay, Ile-de-France, France, ${ }^{4}$ Institute of Atomic and Molecular Sciences, Tapei City, Taipei, Taiwan (Republic of China)

Cathodoluminescence spectroscopy is a powerful technique to study optical properties at the nanoscale. The light emitted by the sample after a local excitation by the electron beam is collected and can be analysed either with a spectrometer [1,2] or an intensity interferometer [3,4]. The first gives access to the spectral properties with a nanometer spatial resolution while the second measures the autocorrelation function of the cathodoluminescence signal $\left(\mathrm{g}^{(2)}\right)$. In this presentation we will focus on the possibility offered by time-resolved cathodoluminescence to probe and study the nitrogen-vacancy atomic defects of diamond.

Indeed, the development of time resolved Cathodoluminescence (TR-CL) enabled the measurement of the lifetime of excited states in semiconductors with a sub-wavelength spatial resolution. It was used for example to measure the influence of stacking faults on the GaN exciton [5], to probe the role of a silver layer on the dynamics of a YAG crystal [6] or to show the influence of stress on the optical properties of $\mathrm{ZnO}$ nanowires [7]. Recently, the first pump-probe cathodoluminescence experiment using diamond revealed the effect of electrons excitation on the nitrogen vacancy color center [8]. These results demonstrate that time-resolved cathodoluminescence is essential to study the correlation between semiconductor optical and structural properties (composition, defects, strain...). While all these pioneering studies were done using a scanning electron microscope, the improvement of the spatial resolution and the combination with other electron based spectroscopy offered by transmission electron microscopes will be a step forward for TR-CL.

In this presentation, we will discuss the first time-resolved cathodoluminescence experiments within a transmission electron microscope. They were performed in a unique microscope, based on a cold-FEG electron gun [9]. This technology allows among other things to reach a spatial resolution of a few nanometers, essential for the study of point defects. In this presentation we will discuss the first TR-CL results (see figure 1), and advantages and drawbacks of such a technology to perform TR-CL and pumpprobe spectroscopy. We will especially discuss the application of such techniques to the study of atomic defect. 

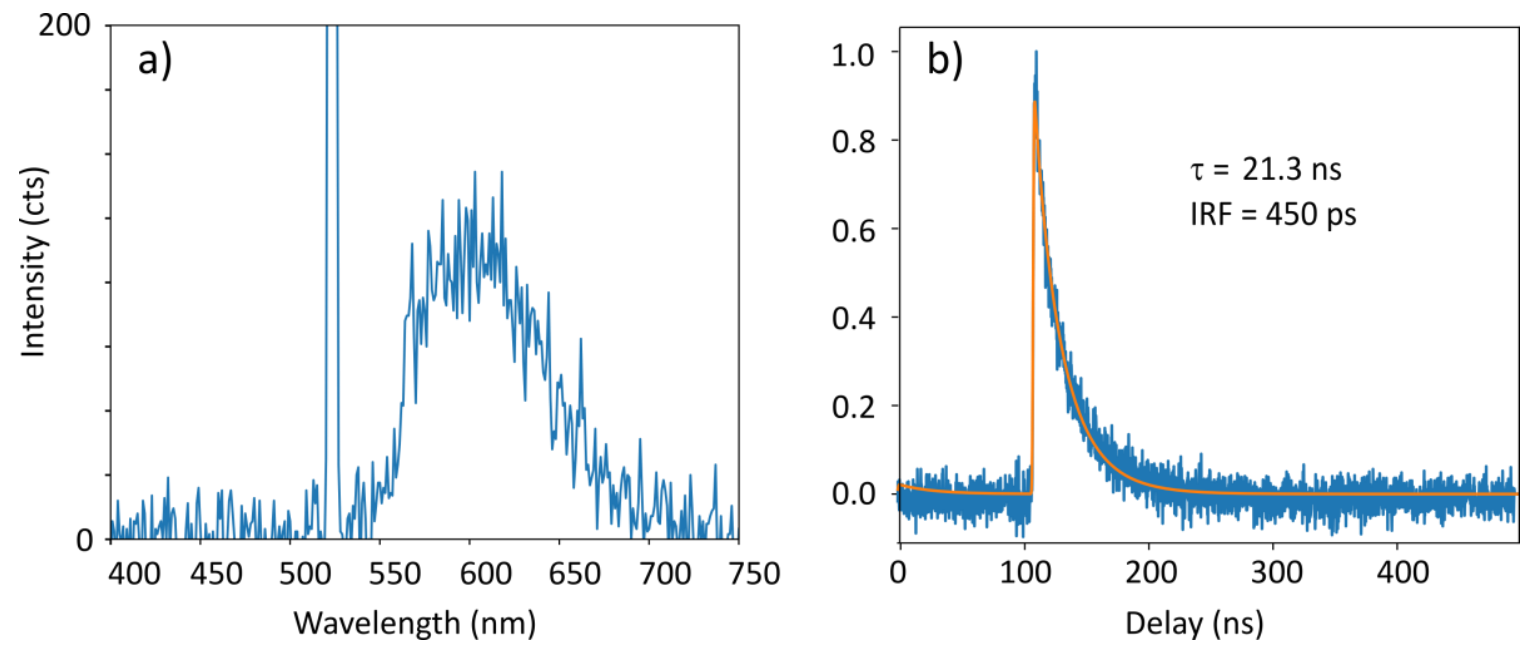

Figure 1. Nitrogen vacancy defect center in Diamond. The spectrum (a) and lifetime (b) were acquired with the pulsed TEM. The high intensity peak at $515 \mathrm{~nm}$ on the spectrum is the laser used to control the electron gun. We found a lifetime of 21,3 ns and an instrumental response function (IRF) of 450 ps due to the resolution of the detection apparatus.

References

1. Yacobi, B. G. \& Holt, D. B. Cathodoluminescence Microscopy of Inorganic Solids. (Springer, 1990).

2. Zagonel, L. F. et al. Nanometer scale spectral imaging of quantum emitters in nanowires and its correlation to their atomically resolved structure. Nano Lett. 11, 568-73 (2011).

3. Tizei, L. H. G. \& Kociak, M. Spatially Resolved Quantum Nano-Optics of Single Photons Using an Electron Microscope. Phys. Rev. Lett. 110, 153604 (2013).

4. Meuret, S. et al. Photon Bunching in Cathodoluminescence. Phys. Rev. Lett. 114, 1-5 (2015).

5. Corfdir, P. et al. Exciton localization on basal stacking faults in a-plane epitaxial lateral overgrown GaN grown by hydride vapor phase epitaxy. J. Appl. Phys. 105, 043102 (2009).

6. Moerland, R. J., Weppelman, I. G. C., Garming, M. W. H., Kruit, P. \& Hoogenboom, J. P. Timeresolved cathodoluminescence microscopy with sub-nanosecond beam blanking for direct evaluation of the local density of states. Opt. Express 24, 24760 (2016).

7. Fu, X. et al. Exciton Drift in Semiconductors under Uniform Strain Gradients: Application to Bent ZnO Microwires. ACS Nano 8, 3412-3420 (2014).

8. Solà-Garcia, M., Meuret, S., Coenen, T. \& Polman, A. Electron-induced state conversion in diamond NV centers measured with pump-probe cathodoluminescence spectroscopy. 0, 20-22 (2019).

9. Houdellier, F., Caruso, G. M., Weber, S., Kociak, M. \& Arbouet, A. Development of a high brightness ultrafast Transmission Electron Microscope based on a laser-driven cold field emission source. Ultramicroscopy 186, 128-138 (2018). 